SHORT REPORTS

\title{
A comparison of $\lg G$ anti-rubella activity in frozen serum stored in primary gel separation tubes or secondary tubes
}

V Ellis, A Charlett, R Bendall

J Clin Pathol 2004;57:104-106

Aims: To determine the suitability of primary gel separation tubes for the storage of frozen sera intended for serological testing.

Methods: Blood samples from 102 patients were collected into gel separation tubes. The sera from these samples were split between the primary gel separation tubes and conventional plastic storage tubes and frozen. A year later, the tubes were thawed and anti-rubella $\lg G$ concentrations were compared for the serum pairs using the Wilcoxon signed rank test.

Results: No significant difference was detected between the two storage methods.

Conclusions: Frozen storage of serum samples in primary gel separation tubes is a practical alternative to storing separated sera in secondary containers. Adopting this practice has advantages for laboratories in reducing specimen handling and reducing errors in labelling stored samples.

$\mathrm{S}$ erum samples are frequently frozen and stored in clinical virology laboratories to permit further testing at a later date. National guidelines ${ }^{1}$ require the storing of antenatal samples for one year and those relating to inoculation injuries for two years. The same document recommends that sera from transplant donors and recipients should be kept for 11 years. Others ${ }^{2}$ have suggested that all serology specimens received by microbiology laboratories should be stored for at least six months. None of these recommendations specifies the type of tube that should be used for storage.

"Serum samples are frequently frozen and stored in clinical virology laboratories to permit further testing at a later date"

Normally, blood is drawn into a primary collection tube, it clots, and any serum required for storage is transferred to a secondary tube, which is labelled and frozen. Small $(1.8 \mathrm{ml})$ screw topped plastic tubes are in general use for this purpose. There are several disadvantages to this system. It is time consuming, it involves staff in extensive contact with potentially infected sera, and introduces the risk of identification errors in relabelling the secondary container. Gel separation tubes have been introduced to simplify the separation of serum from clotted blood. ${ }^{3}$ These tubes contain a plug of a polymer gel and a clot activator. Blood is drawn into the tubes using a vacuum collection system, it clots quickly through the action of the clot activator, and the tube is centrifuged. This results in the separation of the sample into a supernatant layer of serum separated from the clot by an impervious gel plug. The process is carried out in a sealed tube so that no contact with the serum is required. These tubes are used worldwide, mainly for the preparation of serum for biochemical analysis, and have been used in our department for several years.

The purpose of our study was to determine whether sera could be frozen and stored satisfactorily in the primary gel separation tubes. To assess the suitability of this method we compared the antibody activity of gel separated sera stored in the original tube with aliquots of the same sera stored in conventional, secondary tubes. Anti-rubella IgG activity was chosen for our study because it is present at a range of concentrations in the general population, it is often tested on stored sera from antenatal patients, and because accurate, quantitative assays are readily available to measure it.

\section{MATERIALS AND METHODS \\ Patients}

Blood samples for our study were collected from unselected, consecutive adult attendees at the Truro genitourinary medicine clinic. These samples were taken as part of the routine serological screening performed by the department and not specifically for the study.

\section{Blood sampling and processing}

Blood samples were drawn directly into Vacutainer ${ }^{\circledR} 3.5 \mathrm{ml}$ Plus SST (Becton Dickinson, Franklin Lakes, New Jersey, USA) gel separation tubes and sent to the laboratory on the same day. On arrival, the samples were centrifuged and refrigerated overnight. The next morning the serum samples were divided into approximately equal aliquots between the original tube and a screw topped $1.8 \mathrm{ml}$ plastic microtube (Sarstedt, Leicester, UK). These serum pairs were stored at $-20^{\circ} \mathrm{C}$ before testing as part of our study. The original cap was replaced on the SST blood tube for storage.

\section{Serological testing}

Frozen sera were removed from the freezer after 12 months of storage and thawed at room temperature. The thawed sera were tested in matching pairs (SST and microtube fractions of the same original serum) by the VIDAS RUB IgG II (BioMerieux, Marseille, France) automated enzyme linked fluorescent assay, according to the manufacturer's instructions. This automated system reports anti-rubella IgG concentrations over a range of 0 to $400 \mathrm{IU} / \mathrm{ml} .{ }^{4}$ To reduce variability, all paired sera were tested sequentially using kits from the same batch.

\section{Sample size and statistical analysis}

Anti-rubella antibodies are not normally distributed in the adult population, but are skewed to the right. For this reason, a non-parametric test, the Wilcoxon matched pairs signed rank test, was chosen to analyse the results. The analysis was performed both excluding and including those sera for which the anti-rubella IgG concentrations exceeded the upper limit of detection. A right censored regression analysis was used to 
estimate the average difference in the paired samples when including those sera with values above the upper limit of detection. All statistical analyses were performed using Stata 7.0 (Stata Corporation, College Station, Texas, USA).

Using the manufacturer's values for the "within run" coefficient of variability for the VIDAS assay, simulations were carried out using different sample sizes to detect a 5\% difference between the paired serum samples. Using this method, we determined that a sample of at least 86 serum pairs would be required to detect a $5 \%$ difference in antirubella IgG concentrations at a power of $80 \%$.

\section{RESULTS}

The SST tubes were inspected after storage and the gels were all intact. A total of 102 serum pairs were tested after one year in frozen storage. Sixteen serum pairs were initially excluded from analysis, because one or both samples were found to have anti-rubella IgG concentrations greater than the upper limit of detection in the VIDAS assay. Of the remaining 86 samples, the IgG concentrations ranged from 0 to $331 \mathrm{IU} / \mathrm{ml}$ in the SST tubes and 0 to $360 \mathrm{IU} / \mathrm{ml}$ in the microtubes (fig 1). The mean (median) values were 135 (119) and 136 (124) IU/ml, respectively, confirming that the distribution of anti-rubella IgG in this population was positively skewed. Statistical analysis by Wilcoxon sign rank test showed no significant difference between the two groups of stored sera (estimated difference, $-1.5 \mathrm{IU} / \mathrm{ml} ; 95 \%$ confidence interval (CI), -3.5 to $0.5 ; \mathrm{p}=0.18$ ). The estimated difference using all 102 sera samples in the censored regression analysis was $-0.7 \mathrm{IU} / \mathrm{ml}$ (95\% CI, -3.3 to $1.8 ; \mathrm{p}=0.6$ ), which is similar to the non-parametric estimates and not significant.

\section{DISCUSSION}

Our study could not detect a difference in anti-rubella IgG activity between sera stored for a year at $-20^{\circ} \mathrm{C}$ in microtubes or in the original gel separation tube. The SST tube could therefore be used for medium term storage of frozen sera in the original container, at least for the estimation of antirubella IgG. If anti-rubella activity is typical of other IgG antibodies, these sera should also be suitable for other IgG tests. We cannot comment on the suitability of this technique for other antibody classes or other analytes. Others have found that specimens stored in gel separation tubes are suitable for quantitative hepatitis C polymerase chain reaction ${ }^{5}$ estimation, but not for assaying some classes of therapeutic drugs. ${ }^{6}$ In practice, archived sera are usually accessed to look for immunity to infection (for example, to varicella zoster virus in a pregnant women exposed to chicken pox) or to try to establish an onset date for a newly recognised infection (for example, hepatitis C post-inoculation injury). In both cases, it is the detection of IgG in stored sera that is important and the SST tube should be suitable.

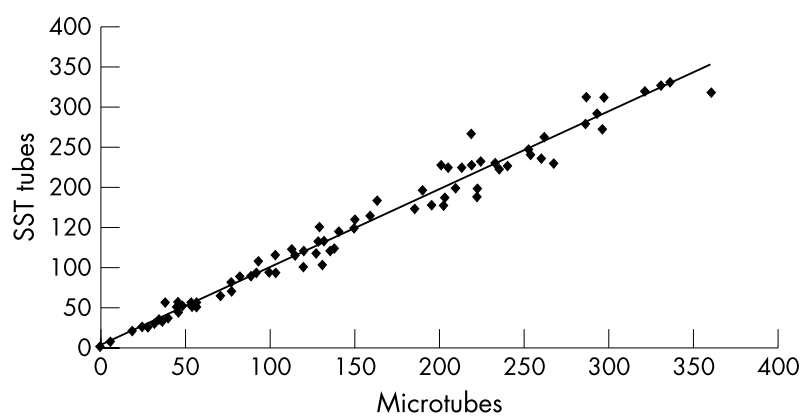

Figure 1 Comparison of anti-rubella lgG concentrations in sera stored in primary SST tubes and microtubes.

\section{Take home messages}

- There were no significant differences in anti-rubella $\lg G$ activity between samples stored frozen for one year in either gel separation tubes or conventional plastic storage tubes

- Thus, frozen storage of serum samples in primary gel separation tubes is a practical alternative to storing separated sera in secondary containers

- Adopting this practice has the advantages of reducing specimen handling, reducing costs, and reducing errors in labelling stored samples

- The main disadvantage is that these tubes require more storage space

- The suitability of this technique for other antibody classes or other analytes would need to be investigated separately

Adopting frozen storage of primary gel separation samples has several advantages to the laboratory. First, the consumable and labour costs associated with serum separation and labelling of storage tubes are eliminated. In our laboratory 20000 sera are stored annually. The savings are $£ 1359$ in consumables (microtubes, labels, and disposable transfer pipettes) and $£ 2692$ in staff time. Set against this, at current prices, the switch from plain Vacutainer tubes to SST tubes costs an extra $£ 754$ each year. From these figures, we estimate the total saving made by our laboratory to be $£ 3297$ each year.

"If anti-rubella activity is typical of other $\lg G$ antibodies, these sera should also be suitable for other lgG tests"

In addition, storing primary SST tubes substantially reduces the risks to laboratory staff associated with handling serum because processing and storing the sample are all done with a sealed tube. The identification of stored sera is more secure because there is no possibility of transcription errors in labelling storage vessels. For some categories of stored sample, such as those taken from the victims of inoculation injuries, the specimen can be collected, separated, and stored without ever being opened.

There are also some disadvantages to this system. For instance, there is no certainty, without extensive testing, that analytes will not react with the gel or tube and decline with time. Although IgG is of most interest to routine laboratories, other classes of antibody and viral antigens such as hepatitis B surface antigen are sometimes of interest, and may behave differently in storage. However, information on storage of these analytes is also lacking for the current system of storage in secondary plastic tubes. The other issue is freezer space. Primary sampling tubes are of necessity larger than the $1.8 \mathrm{ml}$ tubes used routinely for serum storage and do not fit into the racks designed for them. Stored in racks, primary SST tubes occupy more than twice as much space as microtubes (2.16 litre/100 tubes $v 0.89$ litre/100 tubes). This may limit the adoption of frozen storage in primary gel separation tubes by some laboratories. However, overall it is our belief that this method is suitable for medium term storage of sera and solves many of the problems associated with serum storage in routine diagnostic virology laboratories.

\section{Authors' affiliations}

V Ellis, R Bendall, Department of Clinical Microbiology, Royal Cornwall Hospital, Penventinnie Lane, Treliske, Truro, Cornwall TRI 3LQ, UK 
A Charlett, Statistics Unit, Health Protection Agency, Communicable Disease Surveillance Centre, 61 Colindale Avenue, London NW9 5EQ, UK

Correspondence to: Dr R Bendall, Department of Clinical Microbiology, Royal Cornwall Hospital, Penventinnie Lane, Treliske, Truro, Cornwall TR1 3LQ, UK; richard.bendall@cornwall.nhs.uk

Accepted for publication 17 July 2003

\section{REFERENCES}

1 Working Party of the Royal College of Pathologists and the Institute of Biomedical Science. The retention and storage of pathological records and archives. London: Royal College of Pathologists, 1999.
2 Waghorn DJ. Serological testing in a microbiology laboratory of specimens from patients with suspected infectious disease. I Clin Pathol 1995;48:358-63

3 Bush VJ, Janu MR, Bathur F, et al. Comparison of BD Vacutainer SST Plus Tubes with BD SST II Plus Tubes for common analytes. Clin Chim Acta 2001;306:139-43.

4 Vlaspolder F, Singer P, Smit A, et al. Comparison of immulite with vidas for detection of infection in a low-prevalence population of pregnant women in The Netherlands. Clin Diagn Lab Immunol 2001;8:552-5.

5 Grant PR, Kitchen A, Barbara JA, et al. Effects of handling and storage of blood on the stability of hepatitis C virus RNA: implications for NAT testing in transfusion practice. Vox Sang 2000;78:137-42.

6 Karppi J, Akerman KK, Parviainen M. Suitability of collection tubes with separator gels for collecting and storing blood samples for therapeutic drug monitoring (TDM). Clin Chem Lab Med 2000;38:313-20. 